\title{
Eutectic mixture of local anaesthetics (EMLA) 5\% cream as a primary dressing on a painful lower leg ulcer
}

Patients living with chronic leg ulceration may frequently experience moderate

to severe wound-related pain, which may not be alleviated by oral analgesics

alone. Poorly controlled leg ulcer pain can prevent timely and effective

wound management strategies being implemented, and increase wound healing

times. Furthermore, patients with poorly controlled leg ulcer pain can

experience continuous pain, which significantly affects quality of life. This case

report introduces an innovative way of using the eutectic mixture of local

anaesthetics (EMLA) 5\% cream to reduce wound-related pain, reduce oral

analgesic intake, and improve health-related quality of life for a patient with a

painful, chronic lower leg ulcer.

leg ulcer; wound-related pain; eutectic mixture of local anaesthetics (EMLA)

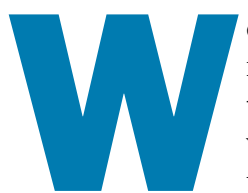

ound-related pain (WRP) has been identified as a major limiting factor in the daily life of patients with chronic leg ulcers. ${ }^{1}$ A study of patients with leg ulcers of venous or mixed aetiology reported that $85 \%$ of participants experienced WRP, with a mean pain score of 4.6 on the numerical rating scale (NRS; range $1-10){ }^{2}$ A score of $\geq 4$ is considered significant pain. ${ }^{3}$

Scores that are persistently $\geq 4$ can indicate uncontrolled WRP, ${ }^{3}$ and WRP may increase significantly during and between dressing changes, and throughout wound debridement. ${ }^{3,4}$ Strategies for reducing WRP are to treat the underlying cause, address local factors such as ischaemia, infection, wound desiccation, excessive exudate, oedema and maceration of the surrounding skin, and to consider analgesic and co-analgesic options. ${ }^{3}$

Recommended analgesic pain intervention generally includes non-opioid analgesia, such as nonsteroidal anti-inflammatory drugs and/or paracetamol, as well as opioids with adjuvant drugs recommended for severe pain. ${ }^{5}$ However, evidence suggests that WRP is frequently under-treated, with many patients taking minimal analgesics or none at all. ${ }^{6}$ Even with appropriate pain management strategies in place, some patients may experience persistent pain at the wound site and/or the surrounding tissues. ${ }^{4}$ This persistent WRP may reduce their ability to tolerate appropriate wound treatment, such as the application of compression therapy, and thus contribute to delayed or reduced wound healing potential. ${ }^{3}$

To date, literature regarding topical analgesic/ anaesthetic treatments for leg ulcer WRP has focused primarily on morphine gel, lignocaine gel, slow release, low-dose ibuprofen in a foam dressing, and the eutectic mixture of local anaesthetic 5\% cream (EMLA). ${ }^{7}$ A recent systematic review found no evidence to support one agent as being most effective in relieving WRP; however, EMLA was found to reduce WRP when applied to the wound bed of chronic leg ulcers before debridement. ${ }^{7}$

The Australia and New Zealand Clinical Practice Guideline for Prevention and Management of Venous Leg Ulcers, ${ }^{8}$ and World Union of Wound Healing Societies (WUWHS) guidelines ${ }^{4}$ currently recommend the use of EMLA for the debridement of venous leg ulcers, but make no recommendation for its use as a primary dressing.
A. Purcell, ${ }^{1,2} \mathrm{RN}, \mathrm{RM}, \mathrm{NP}$, BN, MNurs (Nurs Prac), $\mathrm{PhD}$ candidate, Nurse Practitioner,Wound Management, Central Coast Community Nursing Service;

A. Marshall, ${ }^{3}$ Professor of Acute and Complex Care Nursing,

J. King,' BA(Hons), Clinical Nurse Consultant, Research, Nursing \& Midwifery Directorate; T. Buckley, ${ }^{2} \mathrm{RN}$, BSc(Hons), MN, PhD, Senior Lecturer; I Central Coast Local Health District,Australia; 2 Sydney Nursing School, University of Sydney, Australia;

3 Griffith University and the Gold Coast University Hospital,Australia. Email:apurcell@nsccahs. health.nsw.gov.au 


\section{practice}
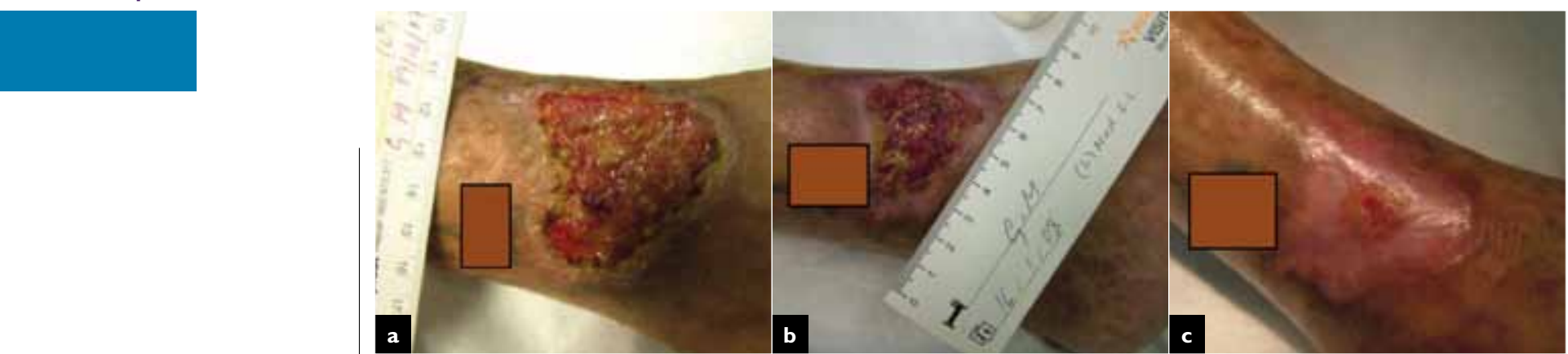

Fig I. Photographs of the wound before application of EMLA $\left(18.54 \mathrm{~cm}^{2} ; \mathrm{a}\right)$, after I week $\left(I I .44 \mathrm{~cm}^{2} ; \mathrm{b}\right)$, and after 12 weeks $\left(0.97 \mathrm{~cm}^{2} ; \mathrm{c}\right)$. For confidentiality, the brown box beside the wound is covering a tattoo

In this case report, we demonstrate how EMLA, when used as a primary dressing on a chronic lower leg ulcer, reduced WRP, improved quality of life, reduced the patient's oral analgesic intake, and did not interfere with the healing potential of the wound, resulting in wound closure.

\section{Case presentation}

A 49-year-old woman with a 9-month history of an extremely painful, right medial lower leg venous leg ulcer (VLU), presented to the nurse practitioner's wound clinic for assessment and implementation of a wound management plan. She was well known to health professionals and was receiving care from a vascular surgeon, neurologist, rheumatologist, general medical practitioner, and community nursing service. She had a history of hypertension, previous intravenous drug use, vasculitic neuropathy developed on the background of hepatitis C, an elevated rheumatoid factor, Raynaud's phenomenon, venous disease and smoking.

The patient presented to the clinic walking on the metatarsal heads of her right foot. During clinical examination, she described the increasing difficulty she was having with daily activities, such as mobilisation, shopping, socialising, working and household tasks, owing to the pain associated with her VLU. She described her pain as sharp, shooting and burning with a pain rating, using the NRS, of 9/10. This resulted in very poor sleep over the previous 9 months. During the clinic visit, the patient appeared debilitated, exhausted and emotional.

The wound surface area was $18.54 \mathrm{~cm}^{2}$. The wound had a low level of exudate, thin, soft yellow-to-brown adhered non-viable tissue covering most of the wound bed, and the wound margins were dry and crusty (Fig 1a). The intact surrounding tissues were highly sensitive to tactile stimuli. There was minimal lower leg oedema and no signs of spreading infection. Several primary dressings had been used previously, including $0.9 \%$ iodine in a starch polymer base ointment, silver Hydrofiber, hydrogel, and silver alginate; however, these dressings did not appear to reduce WRP nor aid wound healing. An absorbent pad initially, followed by silicone foam, had previously been used as the secondary dressings.

The patient continued to experience excruciating, unresolved WRP over a 9-month period. Various analgesic agents were prescribed but had little effect. These included oral ibuprofen, paracetamol/ codeine and oxycodone hydrochloride, plus topical lignocaine gel to the wound bed. Ineffective pain relief resulted in a painful wound that failed to heal and an inability to tolerate optimum wound management strategies, such as compression therapy. Her WRP and the prescribed opiate analgesic medication dulled her ability to function adequately. This was evidenced by her inability to work and socialise. With most treatment options exhausted, an alternative treatment was considered. The topical application of EMLA (EMLA Cream; AstraZeneca Inc.) to the wound bed was trialed as a primary dressing in an effort to provide the patient with effective pain relief.

Her wound was cleansed with normal saline $0.9 \%$. A measured dose of $1 \mathrm{gm}$ of EMLA per $10 \mathrm{~cm}^{2}$ was applied to the right medial lower leg VLU as the primary dressing, with silicone foam continuing as the secondary dressing. Within the first 24 hours, she experienced a marked reduction in WRP from NRS $9 / 10$ to $5 / 10$, and reported having her first full night of sleep in 9 months. The applications of EMLA continued daily. Within 1 week, extra doses of oxycodone hydrochloride necessary before dressing changes were reduced. Within 2 weeks, regular doses of slow-release oxycodone hydrochloride were eliminated altogether and, by week 3 , her WRP level was further reduced (NRS 3/10). The patient also reported a significant improvement in her quality of life following this reduction in WRP and analgesia. She was able to walk with normal action, and shop and socialise more frequently compared with the weeks before commencing EMLA. After 4 weeks, the patient was able to tolerate compression therapy.

Throughout the 13 weeks of EMLA application, there was no evidence of wound or peri-wound maceration. Furthermore, there was no increase in exudate level, bacterial load or non-viable tissue on the wound bed. This, together with compression therapy, resulted in a significant improvement in wound healing time compared with the previous 9 months. Within 1 week after the initial application of EMLA, the wound had decreased in surface area from $18.54 \mathrm{~cm}^{2}$ to $11.44 \mathrm{~cm}^{2}$ (Fig 1b). By week 12, it had reduced to $0.97 \mathrm{~cm}^{2}$ (Fig 1c). After 
13 weeks of daily application of EMLA as the primary dressing, the wound had completely healed. Six months after complete wound closure there has been no recurrence of the wound.

\section{Discussion}

Unresolved pain can negatively impact wound healing in acute and chronic wounds. The causes of leg ulcer pain can be diverse. Pain can be a normal reaction to the inflammatory response to wounding and to damaged nerves. ${ }^{7}$ Oedema, thrombosis, skin changes, ischaemia and inflammation can also result in leg ulcer pain, ${ }^{9}$ with increased non-viable tissue, bacterial burden, wound infection, cellulitis, maceration, wound desiccation, and poor wound management and product selection also contributing factors. ${ }^{7}$

Comprehensive assessment of the patient and the wound are crucial to the identification of pain type and intensity, resulting in timely effective management. It is paramount that the underlying causes are identified and treated and the local factors described above are addressed as soon as possible. ${ }^{3,10}$ If not, WRP reduction strategies may be ineffective.

This novel strategy to reduce WRP in a chronic VLU was initiated following review of the literature to determine the safety of repeated applications of EMLA to a VLU over an extended period of time, and following informed consent by the patient.

Although the literature has reported the use of topical medications, including EMLA, as primary dressings for reducing VLU pain, ${ }^{7,8}$ in this case EMLA was chosen as it is readily available in our health service and the patient had responded well to applications of EMLA before debridement. Morphine gel was not considered as there were no compounding facilities for morphine gel available at the time and access required medical prescribing owing to morphine's opiate status, as this drug was not on the nurse practitioner's approved formulary. Lignocaine gel $2 \%$ was available but was not considered as it had previously had little effect on reducing WRP, and an ibuprofen foam dressing was not available in Australia at the time.

EMLA $5 \%$ cream is a eutectic mixture of $1: 1$ oil/ water emulsion of lignocaine $2.5 \%$ and prilocaine $2.5 \%$ that is non-sterile and preservative free. ${ }^{11}$ When mixed, they form an oil at temperatures above $16^{\circ} \mathrm{C}$, hence a eutectic mixture. ${ }^{11}$ The overall 5\% concentration of EMLA was maintained to reduce systemic toxicity that is linked to higher concentrations. ${ }^{12}$

Lignocaine and prilocaine are both amide-type local anaesthetic agents that stabilise the neuronal membrane, preventing the initiation and conduction of nerve impulses and thereby effecting local anaesthetic action and providing dermal anaesthesia. EMLA applied to intact skin has direct effects on smooth muscle cells, resulting in vasoconstriction, which reaches its peak after $90 \mathrm{~min}$.
During prolonged application, there is pure vasodilatation and increased perfusion in the deeper underlying vessels after $2-3$ hours, ${ }^{13}$ hence no negative effect on skin blood flow. ${ }^{13-15}$ EMLA on a leg ulcer over a 24-hour period has been found to be systemically safe. ${ }^{16}$ Furthermore, it has been reported that, even after 24 hours of continuous exposure with up to $10 \mathrm{~g}$ of EMLA on a leg ulcer, there was no evidence of neurotoxicity. ${ }^{16}$

One study determined that the peri-operative application of EMLA in the breast and axilla area reduced analgesic requirements, as well as the acute and chronic pain after breast surgery. ${ }^{17}$ EMLA was also found to have rapid acting and powerful antibacterial effects in vitro on typical wound pathogens, such as Staphylococcus aureus, Escherichia coli, Pseudomonas aeruginosa and Streptococcus pyogenes, suggesting some benefit in bacteria minimisation is possible. ${ }^{18}$ Furthermore, EMLA has also been found to significantly increase cell multiplication; ${ }^{19}$ however, the effect EMLA has on wound healing is unknown. ${ }^{7}$

The principle use of EMLA is to provide dermal anaesthesia. ${ }^{11}$ EMLA cream is currently used before IV catheter insertion, blood sampling, vaccination, superficial surgical procedures, as well as for topical anaesthesia of leg ulcers during cleansing and wound

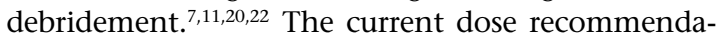
tions for the use of EMLA before debridement is $1-2 \mathrm{~g} / 10 \mathrm{~cm}^{2}$, up to a total of $10 \mathrm{~g}$, with an application time of $30-60 \mathrm{~min} .{ }^{11}$ The depth and quality of the anaesthesia depends on the characteristics of the ulcer, application time and the dose applied. ${ }^{11}$

For this patient who had a VLU, the use of EMLA was effective in significantly reducing WRP. It was evident that this rapid, effective reduction of WRP was also accompanied by a reduction in oral opiate analgesic intake, thus minimising any side effects of these medications. Furthermore, optimum wound management strategies, such as compression therapy, were instigated 1 month after the commencement of the daily application of EMLA to the wound bed, which had been previously not possible. Wound healing was evident within the first week of the daily application of EMLA, even before the application of compression therapy, suggesting no detriment to wound healing following repeated daily applications of this anaesthetic cream. For this patient, EMLA was well tolerated with no adverse reactions to the medication detected.

However, adverse reactions to EMLA have been reported in the literature, although these are usually transient and localised. The most frequently observed adverse reactions include blanching, erythema and oedema. Less frequently, pruritus, burning, purpura and contact hypersensitivity have been reported. ${ }^{11}$ Methemoglobinaemia, although rare, is an important systemic concern regarding 
EMLA, particularly in neonates and young children, ${ }^{11,22}$ thus EMLA is not recommended in patients under 3 months of age. ${ }^{23}$ One report highlighted a particular patient having seizures following the seventeenth application of EMLA for the debridement of a Martorelle's ulcer; however, a causal link to the use of EMLA was not established. ${ }^{24}$ The authors recommend caution when applying EMLA to elderly compromised skin.

\section{Conclusion}

This case report demonstrates how a patient with a painful chronic venous lower leg ulcer, who had not responded favourably to conventional woundrelated pain management strategies, responded very well to the daily application of EMLA cream $5 \%$ to the wound bed, with a significant and rapid reduction of WRP, oral analgesia and wound size to complete healing, coupled with improved healthrelated quality of life.

At time of publication, no studies have reported using EMLA on chronic leg ulcers as a primary dressing to control WRP. To the best of our knowledge, this is the only case reported in the literature. Further investigation of this novel strategy is warranted. A randomised control trial is currently in progress by the authors to test the hypotheses that the daily topical application of EMLA 5\% cream to the wound bed of painful chronic leg ulcers, as the primary dressing, will reduce WRP and analgesia requirements. Secondary outcome measures will be improved health-related quality of life and decreased wound healing times for patients with painful chronic leg ulcers.

\section{References}

I Persoon,A., Heinen, M.,Van der Vleuten, $C$. et al. Leg ulcers: a review of their impact on daily life. J Clin Nurs. 2004; I 3: 3, 34I-354.

2 Heinen, M., Persoon, A., Van de Kerkhof, P. et al. Ulcer-related problems and health care needs in patients with venous leg ulceration:A descriptive cross-sectional study. Int J Nurs Studies. 2007; 44: 8, I 296-1 303.

3 World Union of Wound Healing Societies. Principles of Best Practice: Minimising Pain at Wound Dressing-Related Procedures. A Consensus Document. MEP Ltd, 2004.

4 World Union of Wound Healing Societies. Minimizing Pain at Wound Dressing-Related Procedures: Implementation of Pain Relieving Strategies. MEP Ltd, 2007. 5 World Health Organization. WHO's Pain Ladder. c20II. Available from: http://tinyurl. com/64pp2t [Accessed June 20I2]. 6 Woo, K.Y., Sibbald, G. Fogh, K. et al.Assessment and management of persistent (chronic) and total wound pain. Int Wound J. 2008; 5 : 2, I44-I57.

7 Briggs, M., Nelson, E.A.Topical agents or dressings for pain in venous leg ulcers. Cochrane Database Syst Rev. 2010; 4: CD00II77.
8 The Australian Wound Management Association Inc., The New Zealand Wound Care Society. Australia and New Zealand Clinical Practice Guideline for Prevention and Managment of Venous Leg Ulcers [Draft]: Cambridge Publishing, 20I I Available from: http://tinyurl.com/ chp7du6 [Accessed June 2012]. 9 Krasner, D.L., Rodeheaver, G.T., Sibbald, R.G. (eds). Chronic Wound Care:A Clinical Source Book for Health Care Professionals (4th edn). HMP Communications, 2007. 10 Woo, K.Y., Harding, K., Price, P., Sibbald, R.G. Minimising wound-related pain at dressing change: evidence-informed practice. Int Wound J. 2008; 5: 2 , I44-157.

I I AstraZeneca Canada Inc. EMLA Cream. Product

Monograph. 2012. Available from: http://tinyurl.com/7ul8avj [Accessed June 2012].

I 2 Friedman, P., Mafong, E., Friedman, B., Geronemus, R. Topical anaesthetics update: EMLA and beyond. Dermatol Surg. 200 I; 27: I2, 1019-1026. 13 Bjerring, P.,Andersen, P., Arendt-Nielsen, L.Vascular response of human skin after analgesia with EMLA cream. $\mathrm{Br}$ J Anaesth. 1989; 63: 6, 655-660.
I 4 Hafner, H., Thomma, S., Eichner, $M$. et al. The influence of EMLA cream on cutaneous circulation. Clin Hemorheol Microcirc. 2003; 28: 3, $121-128$.

15 Wiles, M.D., Dobson, S.A., Moppett, I.K.The effect of a new topical local anaesthetic delivery system on forearm skin blood flow reactivity. Anaesthesia. 2010 65: 2, 178-183.

16 Stymne, B., Lillieborg, S. Plasma concentrations of lignicaine and prilocaine after a 24-hr application of analgesia cream (EMLA) to leg ulcers. Br J Dermatol. 200I; I45: 4, 530-534.

17 Fassoulaki,A., Sarantopoulos, C., Melemeni,A., Hogan, Q. EMLA reduces acute and chronic pain after breast surgery for cancer. Reg Anesth Pain Med. 2000; 25: 4, 350-355.

I 8 Berg, J.O., Mossner, B.K., Skov, M.N. et al.Antibacterial properties or EMLA and lidocaine in wound tissue biopsies for culturing. Wound Repair Regen. 2006; | 4: 5, 58|-585.

19 O'Brien, M.C., Breuning, E. Webb,J. et al.A prospective randomised controlled trial to investigate the effect of local anaesthetic in vivo on cell structure. J Plast Reconstr Anaesthet Surg. 2008; 61: 10, 1232-1234.
20 Blanke, W., Hallern, B. Sharp wound debridement in local anaesthesia using EMLA cream: 6 years' experience in 1084 patients. Eur J Emerg Med. 2003; 10: 3, 229-231.

2I Vanscheidt,W., Sadjadi, Z., Lillieborg, S. EMLA anaesthetic cream for sharp leg ulcer debridement: a review of the clinical evidence for analgesia efficacy and tolerability. Eur J Emerg Med. 200 I; II: 2, 90-96. 22 Friedman, P., Mafong, E.,

Freidman, E., Geronemus, R. Topical anaesthetic update: EMLA and beyond. Dermatol Surg. 200 I; 27: 12, 1019-1026.

23 Crystal, C., McArthur, T., Harrison, B. Anesthetic and procedural sedation techniques for wound management. Emerg Med Clin North Am. 2007; 25: I, 4I-7I.

24 Boulinguez, S., Sparsa, A., Bouyssou-Gauthier, M.Adverse effects associated with EMLA cream used as topical anaesthetic for the mechanical debridement of leg ulcers. J Am Acad Dermatol. 2000; 42: I Pt I, |46-| 48.

\section{Find out more about the JWC, subscribe}

and submit articles at: www.journalofwoundcare.com 